\section{A atual e necessária proteção do trabalhador: câncer e ocupação}

The current and mandatory protection of the worker: cancer and occupation

Eliane de Freitas Drumond

(https:// orcid.org/0000-0003-2159-4250) ${ }^{1}$

Jussara de Medeiros Silva

(https://orcid.org/0000-0002-2825-7006) ${ }^{2}$

Paulo Guilherme de Oliveira Salles

(https://orcid.org/0000-0001-8839-3491) ${ }^{1}$

${ }^{1}$ Núcleo de Ensino e Pesquisa do Instituto Mário Penna. Belo Horizonte MG Brasil.

${ }^{2}$ Gerência de Saúde do Trabalhador, Secretaria Municipal de Saúde. Belo Horizonte MG Brasil.

Prezados editores,

Neoplasias malignas de pênis afetam principalmente indivíduos de baixo nível socioeconômico e escolaridade, residentes em países em desenvolvimento. Coelho et al. ${ }^{1}$ relataram que residentes em zonas rurais do Maranhão tiveram maior dificuldade de acesso ao tratamento.

O manuscrito "Análise de sobrevida de pacientes com câncer de pênis tratados em um hospital terciário de oncologia" ${ }^{2}$ traz interessantes resultados sobre os quais julgamos necessárias algumas considerações. É digno de nota o excelente preenchimento da ocupação. Porém o critério adotado para classificar a ocupação como rural e não rural não está explicitado na metodologia. Pode-se supor que tenha sido adotada como proxy de situação socioeconômica, reconhecidamente pior entre residentes em áreas rurais brasileiras. Surpreende o resultado da utilização da ocupação rural e não rural na análise de sobrevida: menor sobrevida dos trabalhadores não rurais residentes na Grande Vitória, diferentemente do observado na literatura ${ }^{1,3,4}$ onde se vê que trabalhadores rurais são mais expostos a carcinogênicos e têm piores condições socioeconômicas e de acesso aos serviços de saúde, relacionados ao pior prognóstico do câncer.

O Registro Hospitalar de Câncer (RHC) é importante fonte de análise sobre câncer, mas nele, como em outras bases de dados, se observa elevado percentual de incompletude da ocupação ${ }^{4,5}$.
Estabelecido em Belo Horizonte, o Instituto Mário Penna, integrado pelos Hospitais Mário Penna e Luxemburgo, é referência mineira na assistência aos pacientes com câncer e no seu âmbito encontra-se em desenvolvimento projeto intitulado "Avaliação da qualidade do RHC do Instituto Mário Pena”. Para possibilitar análise envolvendo ocupação e morte por câncer os autores dessa carta realizaram relacionamento determinístico entre RHC e Sistema de Informação sobre Mortalidade (SIM). Os 1.273 óbitos hospitalares por câncer ocorridos em 2016 e 2017 foram investigados em prontuários e pareados ao SIM (resultado não publicado). Manteve-se elevado (56\%), no entanto, o percentual de ocupações como dona de casa e aposentado. Apesar de sua importância, falhas no preenchimento da ocupação nos sistemas de informação em saúde brasileiros ainda geram prejuízo às análises sobre a relação entre trabalho e câncer.

Incentivos ao preenchimento correto e consistente assim como à adequada descrição da metodologia de utilização da ocupação são fundamentais para que análises feitas pelo serviço de saúde e pela academia possam contribuir com a melhoria dos instrumentos de vigilância e proteção do trabalhador, especialmente em cenário de perda de direitos.

\section{Referências}

1. Coelho RWP, Pinho JD, Moreno JS, Garbis DVO, Nascimento AMT, Larges JS, Calixto JRR, Ramalho, LNZ, Silva AAM, Nogueira LR, Moura Feitosa L, Silva GEB. Penile cancer in Maranhão, Northeast Brazil: the highest incidence globally? BMC Urol 2018; 18(1):50.

2. Souza MAC, Zacchi SR, Viana KCG, Souza CB, Zandonade E, Amorim MHC. Survival analysis of penile cancer patients treated at a tertiary oncology hospital. Cien Saude Colet 2018; 23(8):2479-2486.

3. Reis AA, Barcelos PL, Peclat PAA, Saddi VA, Cruz AD. Aspectos clínico-epidemiológicos associados ao câncer de pênis. Cien Saude Colet 2010; 15(Supl. 1):1105-1111.

4. Renna JN, Silva GA. Tendências temporais e fatores associados ao diagnóstico em estágio avançado de câncer do colo uterino: análise dos dados dos registros hospitalares de câncer no Brasil, 2000-2012. Epidemiol. Serv. Saúde 2018; 27(2):e2017285.

5. Grabois MF, Souza MC, Guimarães RM, Otero UB. Completude da Informação Ocupação nos Registros Hospitalares de Câncer do Brasil: Bases para a Vigilância do Câncer Relacionado ao Trabalho. Revista Brasileira de Cancerologia 2014; 60(3):207-214. 
